\title{
Characteristics of Patients with Chronic Obstructive Pulmonary Disease Exposed to Different Environmental Risk Factors: A Large Cross-Sectional Study
}

This article was published in the following Dove Press journal:

International Journal of Chronic Obstructive Pulmonary Disease

Jia-xi Duan, ${ }^{1,2}$ Wei Cheng, (iD) ${ }^{1,2}$ Yuqin Zeng, ${ }^{1,2}$ Yan Chen, ${ }^{1,2}$ Shan Cai, ${ }^{1,2}$ Xin Li, iD ${ }^{3}$ Yingqun Zhu, ${ }^{4}$ Ming Chen, ${ }^{5}$ Meiling Zhou, ${ }^{6}$ Li-bing Ma, ${ }^{7}$ Qi-mi Liu, ${ }^{8}$ Ping Chen ${ }^{1,2}$

'Department of Pulmonary and Critical Care Medicine, The Second Xiangya Hospital, Central South University, Changsha, Hunan 4I00II, People's Republic of China; ${ }^{2}$ Research Unit of Respiratory Disease, Central South University, Changsha, Hunan 4I00II, People's Republic of China; ${ }^{3}$ Division 4 of Occupational Diseases, Hunan Prevention and Treatment Institute for Occupational Diseases, Changsha, Hunan 4I0000, People's Republic of China; ${ }^{4}$ Department of Respiratory Medicine, The Third Hospital of Changsha, Changsha, Hunan 4I00II, People's Republic of China; ${ }^{5}$ Department of Respiratory Medicine, The No.I Traditional Chinese Medicine Hospital in Changde, Changde, Hunan 4I5000, People's Republic of China; ${ }^{6}$ Department of Respiratory Medicine, The First People's Hospital of Huaihua, Huaihua, Hunan 4I8000, People's Republic of China; ${ }^{7}$ Department of Respiratory Medicine, Affiliated Hospital of Guilin Medical University, Guilin, Guangxi 541000, People's Republic of China; ${ }^{8}$ Department of Respiratory and Critical Care Medicine, The Second People's Hospital of Guilin, Guilin, Guangxi 541000, People's Republic of China

Correspondence: Ping Chen Department of Pulmonary and Critical Care Medicine, The Second Xiangya Hospital, Central South University, 139 Renmin Middle Road, Changsha, Hunan 41001 I, People's Republic of China Tel +8673185295248

Email pingchen073।@csu.edu.cn
Purpose: Tobacco smoking, biomass smoke, and occupational exposure are the main risk factors for chronic obstructive pulmonary disease (COPD). The present study analyzes data on exposure to these factors in a cohort of patients with COPD and assesses their differences in demographic and clinical characteristics.

Patients and Methods: The cross-sectional observational study was conducted from November 2016 to December 2019. Inclusion criteria were patients aged over 40 years old with post-bronchodilator forced expiratory volume in 1 second $\left(\mathrm{FEV}_{1}\right) /$ forced vital capacity (FVC) <0.7. At baseline, demographic features and exposure history were recorded. Moreover, respiratory symptoms were assessed by the COPD Assessment Test (CAT) and modified Medical Research Council scale (mMRC). A generalized linear mixed model was used to adjust for potential confounders.

Results: A total of 5183 patients with COPD were included in the final analysis. The results demonstrate that exposure to tobacco combined with other risk factors resulted in significantly higher CAT scores $(16.0 \pm 6.7$ vs $15.3 \pm 6.3, P=0.003)$ and more severe dyspnea (patients with $\mathrm{mMRC} \geq 2,71.5 \%$ vs $61.6 \%, P<0.001$ ) than exposure to tobacco alone. In addition, COPD patients with biomass smoke exposure alone had higher CAT scores than patients with only tobacco or occupational exposure (17.5 \pm 6.3 vs $15.3 \pm 6.3$, and $15.2 \pm 6.3$, respectively, $P<0.05$ for each comparison) and were more likely to be female and older. In addition, COPD patients who suffered from occupational exposure developed more severe dyspnea than those exposed to tobacco alone $(70.8 \%$ vs $61.6 \%, P<0.05)$, as did those exposed to biomass smoke alone $(74.2 \%$ vs $61.6 \%, P<0.05)$. This difference remained strong even after adjustment for potential confounders.

Conclusion: There are significant demographic and clinical differences among COPD patients with tobacco smoking, biomass smoke, and occupational exposures.

Keywords: chronic obstructive pulmonary disease, tobacco, biomass smoke, occupational exposure

\section{Introduction}

Chronic obstructive pulmonary disease (COPD), characterized by persistent airflow limitation and progressive respiratory symptoms, is the third leading cause of death worldwide following cardiovascular and cerebrovascular disease. ${ }^{1}$ According to estimates from the Global Burden of Disease Study, more than 174.5 million people suffer from COPD worldwide. ${ }^{2}$ The prevalence among the general Chinese 
population aged 40 years or older has been estimated at $13.7 \%{ }^{3}$ Since COPD is caused by significant exposure to harmful particles and gases, the identification and reduction of deleterious exposures are essential.

Tobacco smoking is the most frequently identified risk factor for COPD. However, many other environmental risk factors likely influence the development of COPD, such as biomass smoke and occupational exposure. The term biomass refers to the energy generated from the combustion of organic products consisting of animal manure, wood, and crop residues. Inhalation of combustion products causes COPD. This exposure is common in regions where biomass is used as a fuel for heating and cooking in poorly ventilated dwellings. ${ }^{4,5}$ A series of studies have elucidated that workplace exposure to dust, gases, coal, or volatile chemicals is responsible for COPD-related outcomes. ${ }^{6,7}$ However, it is sometimes found that patients exposed to occupational hazards or biomass smoke also smoke. It is difficult to disentangle the effects of overlapping exposures on the development of COPD. In this situation, occupational and biomass smoke exposure may be considered additive risk factors for COPD. ${ }^{8}$

Numerous studies around the world have attempted to identify the pathogenesis and pathological changes of COPD caused by tobacco smoking, biomass smoke, and occupational exposures. $^{9-12}$ Until now, few large-scale studies have focused on the symptoms of COPD patients exposed to these different risk factors. Over the past decade, entire occupations and levels of socioeconomic status have transformed, and lifestyles have changed. In this new context, a comparison of the characteristics of COPD with different exposures is essential for the development of targeted prevention programs and rational planning of healthcare resources. Therefore, the goal of this study is to specifically investigate and compare the characteristics of COPD patients exposed to different risk factors.

\section{Patients and Methods}

\section{Study Design and Population}

This was a cross-sectional observational study conducted from November 2016 to December 2019 that enrolled COPD patients in the outpatient department from 12 hospitals in Southern China (Table S1). This study was performed following the ethics principles of the Declaration of Helsinki and was approved by the Ethics Committee of the Second Xiangya Hospital of Central South University in Changsha, Hunan. Written informed consent was obtained from all participants in this trial. This study was registered on the Chinese Clinical Trial Registry (ChiCTRPOC-17010431, http://www.chictr.org.cn/).

All included patients were tested with spirometry and met the diagnostic criteria of COPD (spirometry with a ratio of the forced expiratory volume in 1 second to the forced vital capacity $\left(\mathrm{FEV}_{1} / \mathrm{FVC}\right)$ lower than 0.70 after bronchodilator administration). In addition, all patients were aged over 40 years and able to provide an accurate exposure history. Patients were excluded from the study if they had other chronic respiratory diseases, such as asthma (clinically diagnosed and reversibility $>12 \%$ ), bronchiectasis (based on high-resolution computed tomography), pulmonary fibrosis, tuberculosis, and lung cancer (based on actual diagnoses from case records).

\section{Measurement}

All eligible patients were assessed face-to-face by trained interviewers. Assessments included age, sex, body mass index (BMI), marital status, education level, number of COPD deteriorations in the past 12 months, and lung function measured by spirometry. Tobacco smoking was assessed by cumulative cigarette pack-years. Smokers were people who have smoked equal to or more than 10 pack-years in their lifetime; otherwise, the patients were classified as nonsmokers. We defined biomass smoke exposure as using biomass fuels (wood, grass, charcoal, and crop residues) for cooking or heating at least $2 \mathrm{~h}$ per day for at least 1 year. We defined occupational exposure as exposure to dust, gases/fumes, insecticides, chemical substances, paints, and metals at work for at least 8 hours per day for more than 1 year. $^{13-15}$

The COPD Assessment Test (CAT), a validated eightquestion health-status instrument, includes cough, phlegm, chest tightness, shortness of breath, limited at home, confidence leaving home, sound sleep, and energy, with scores ranging from 0 to $5 .{ }^{16}$ The total score is calculated by simply adding all scores, with higher scores indicating greater symptom severity. ${ }^{17}$ Dyspnea was quantified by using the modified Medical Research Council scale (mMRC, with scores ranging from 0 to 4$).{ }^{18}$ We used an mMRC score of 2 as a threshold for moderate to severe dyspnea. Exacerbation was defined as an acute increase in respiratory symptoms that resulted in additional therapy. Patients were also classified into one of four GOLD 2017 categories $(\mathrm{ABCD})$ based on the intensity of respiratory symptoms (CAT) and previous exacerbations. 


\section{Subgroup Definition}

All COPD patients were divided according to predefined groups. First, we categorized recruited COPD patients into smokers and nonsmokers. Then, we subcategorized the members of each of these two groups into five mutually exclusive groups. The following criteria were used to distinguish different subgroups: 1) COPD patients were exposed to tobacco alone; 2) COPD patients were exposed to tobacco combined with other risk factors including biomass smoke and occupational exposure; 3 ) COPD patients were exposed to biomass smoke alone; 4) COPD patients were exposed to occupational exposure alone; and 5) COPD patients were exposed to other risk factors except tobacco, biomass smoke, and occupational exposure.

\section{Data Analysis}

Categorical variables are summarized as frequencies, while continuous variables were tested for normality and are presented as the mean \pm standard deviation (SD) or the median \pm interquartile range (IQR) in case of skewed data. Chi-square or Fisher's test was used to compare categorical variables. Parametric tests (Student's $t$-test or ANOVA) were used to compare values with a normal distribution and non-parametric tests (Mann-Whitney $U$-test or Kruskal-Wallis test) for data without a normal distribution. Generalized Linear mixed model was performed to control for potential confounders, such as sex, age, education level, body mass index, and marital status. All tests were two-tailed, A P value of less than 0.05 was considered statistically significant. The Statistical Package for Social Sciences 22.0 (IBM Corporation, Armonk, NY, USA) was used for all statistical analyses.

\section{Results}

During the study period, 5424 patients diagnosed with COPD were consecutively recruited, of whom 5183 (96\%) fulfilled all inclusion and exclusion criteria. The mean age was $63.3 \pm 8.7$ years, and $87.4 \%$ of the patients were male. A total of 3688 (71\%) patients were smokers, and 1495 (29\%) patients were nonsmokers (Figure 1). Smokers were older and more likely to be male. They had a higher education level and significantly worse lung function in terms of the predicted $\mathrm{FEV}_{1} \%$ than nonsmokers. In contrast, nonsmokers had higher CAT scores $(16.2 \pm 6.4$ vs $15.7 \pm 6.5$, $P=0.007$, Table 1) than smokers. After sex, age, education, and BMI adjustment, the difference in CAT scores between the two groups remained significant (Table S2). Compared with the smokers, nonsmokers had elevations in several components of the CAT scores, such as confidence leaving home, sleep, and energy (Figure S1).

Among smokers with COPD, 2168 patients were exposed to tobacco alone, and 1520 patients were exposed to tobacco combined with other risk factors including biomass smoke and occupational exposure. We found that patients who were exposed to tobacco combined

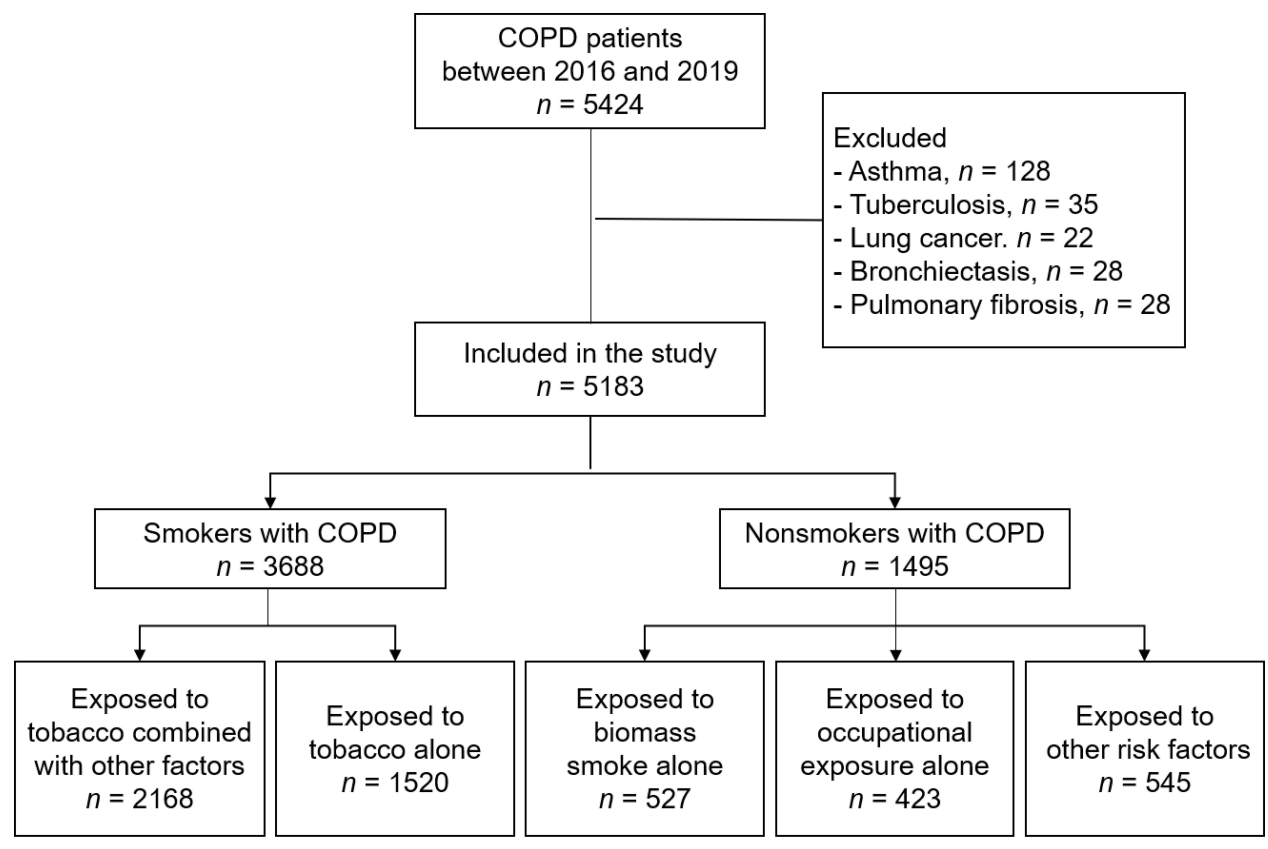

Figure I Study flow diagram.

Abbreviation: COPD, chronic obstructive pulmonary disease. 
Table I Characteristics of the Studied COPD Patients

\begin{tabular}{|c|c|c|c|c|}
\hline & $\begin{array}{l}\text { Total } \\
(n=5 \mid 83)\end{array}$ & $\begin{array}{l}\text { Smokers } \\
(n=3688)\end{array}$ & $\begin{array}{l}\text { Nonsmokers } \\
(n=1495)\end{array}$ & $P$ value \\
\hline Age (years), mean (SD) & $63.3(8.7)$ & $63.7(8.2)$ & $62.2(9.7)$ & $<0.001^{*}$ \\
\hline Sex, N (\%) & & & & $<0.00 I^{*}$ \\
\hline Male & $4528(87.4)$ & $3643(98.8)$ & $885(59.2)$ & \\
\hline Female & $655(12.6)$ & $45(1.2)$ & $610(40.8)$ & \\
\hline BMI (kg/m2), N (\%) & & & & 0.272 \\
\hline$<24$ & $3542(68.3)$ & $2537(68.8)$ & $1005(67.2)$ & \\
\hline$\geq 24$ & $|64|(3 \mid .7)$ & $|I 5|(31.2)$ & $490(32.8)$ & \\
\hline Education, N (\%) & & & & $<0.00 I^{*}$ \\
\hline Middle school or less & $4168(80.4)$ & $2886(78.3)$ & $1282(85.8)$ & \\
\hline High school or upper & $1015(19.6)$ & $802(21.7)$ & $213(14.2)$ & \\
\hline Marital status, N (\%) & & & & 0.840 \\
\hline Unmarried & $43(0.8)$ & $30(0.8)$ & $13(0.9)$ & \\
\hline Married & $5140(99.2)$ & $3658(99.2)$ & $1482(99.1)$ & \\
\hline CAT, mean (SD) & $15.8(6.5)$ & $15.7(6.5)$ & $16.2(6.4)$ & $0.007^{*}$ \\
\hline mMRC, N (\%) & & & & 0.646 \\
\hline $0-1$ & $1678(32.4)$ & $|20|(32.6)$ & $477(31.9)$ & \\
\hline $2-4$ & $3505(67.6)$ & $2487(67.4)$ & $1018(68.1)$ & \\
\hline Previous exacerbations, $\mathrm{N}$ (\%) & & & & 0.127 \\
\hline$<2$ & $3259(62.9)$ & $2343(63.5)$ & $916(61.3)$ & \\
\hline$\geq 2$ & $1924(37.1)$ & $1345(36.5)$ & 579 (38.7) & \\
\hline FEV (liter), median (IQR) & $1.08(0.75)$ & $1.10(0.75)$ & $\mathrm{I} .08(0.7 \mathrm{I})$ & $<0.00 I^{*}$ \\
\hline $\mathrm{FEV}, \%$ predicted (\%), median (IQR) & $44.5(28.0)$ & $43.2(27.9)$ & $45.0(27.5)$ & $<0.00 I^{*}$ \\
\hline FVC (liter), median (IQR) & $2.40(1.04)$ & $2.49(0.99)$ & $2.33(\mathrm{I} .1 \mathrm{I})$ & $<0.00 I^{*}$ \\
\hline FVC\% predicted (\%), median (IQR) & $67.4(26.5)$ & $64.9(24.2)$ & $72.3(33.5)$ & $0.030 *$ \\
\hline $\mathrm{FEV}_{1} / \mathrm{FVC}(\%)$, median (IQR) & $47.1(18.5)$ & $46.0(18.6)$ & $48.6(18.6)$ & $<0.001 *$ \\
\hline$A B C D$ group, $N(\%)$ & & & & $0.023 *$ \\
\hline A & $705(13.6)$ & $530(14.4)$ & $175(11.7)$ & \\
\hline B & $2554(49.3)$ & $1813(49.2)$ & $74 I(49.6)$ & \\
\hline C & $198(3.8)$ & $148(4.0)$ & $50(3.3)$ & \\
\hline $\mathrm{D}$ & $1726(33.3)$ & $1197(32.4)$ & $529(35.4)$ & \\
\hline LAMA, N (\%) & $1508(29.3)$ & $1074(29.1)$ & $434(29.0)$ & 0.948 \\
\hline ICS+LABA, N (\%) & $826(15.9)$ & $607(16.5)$ & $219(14.6)$ & $0.107^{*}$ \\
\hline ICS+LABA+LAMA, N (\%) & $2249(43.4)$ & $1590(43.3)$ & $659(44.1)$ & 0.622 \\
\hline
\end{tabular}

Notes: Categorical variables are summarized by frequencies, and statistical significance was determined by the Chi-square test. Continuous variables are described by mean \pm standard deviation (SD) or median \pm interquartile range (IQR), and statistical significance was determined by Student's $t$-test or Mann-Whitney $U$-test. $* P<0.05$. Abbreviations: BMI, body mass index; CAT, COPD Assessment Test; FEV, forced expiratory volume in I second; FVC, forced vital capacity; ICS, inhaled steroids; mMRC, modified Medical Research Council scale; LABA, long-acting $\beta_{2}$ agonists; LAMA, long-acting muscarinic antagonists.

with other risk factors had a greater chance of acute exacerbation ( $41.5 \%$ vs $29.3 \%, P<0.001$, Table 2$)$ than those in the group exposed to tobacco smoke alone. Higher mean CAT scores $(16.0 \pm 6.7$ vs $15.3 \pm 6.3, P=0.003)$ and more severe dyspnea (patients with $\mathrm{mMRC} \geq 2,71.5 \%$ vs $61.6 \%, P<0.001)$ were also found in patients who were exposed to tobacco combined with other risk factors. More specifically, they had more severe chest tightness and more significantly impacted sleep (Figure S2). The main potential confounders were age and education level. After adjusting for covariates in the generalized linear mixed model, the acute exacerbation frequency $(P<0.001$, Table 4), CAT scores $(P=0.008)$, and mMRC scores $(P<0.001)$ of patients exposed to tobacco combined 
Table 2 Comparison of the Characteristics in the Subgroups of COPD Patients Exposed to Tobacco Alone and Tobacco Combined with Other Risk Factors

\begin{tabular}{|c|c|c|c|}
\hline & $\begin{array}{l}\text { Tobacco Alone } \\
(n=1520)\end{array}$ & $\begin{array}{l}\text { Tobacco Combined with } \\
\text { Other Risk Factors } \\
(n=2168)\end{array}$ & $P$ value \\
\hline Age (years), mean (SD) & $64.6(8.2)$ & $63.2(8.1)$ & $<0.001 *$ \\
\hline $\begin{array}{l}\text { Sex, } N(\%) \\
\text { Male } \\
\text { Female }\end{array}$ & $\begin{array}{l}1498(98.6) \\
22(1.4)\end{array}$ & $\begin{array}{l}2145(98.8) \\
23(1.2)\end{array}$ & 0.293 \\
\hline $\begin{array}{l}\text { BMl }(\mathrm{kg} / \mathrm{m} 2), \mathrm{N}(\%) \\
\quad<24 \\
\quad \geq 24\end{array}$ & $\begin{array}{l}1024(67.4) \\
496(32.6)\end{array}$ & $\begin{array}{l}1513(69.8) \\
655(30.2)\end{array}$ & 0.119 \\
\hline $\begin{array}{l}\text { Education, N (\%) } \\
\text { Middle school or less } \\
\text { High school or upper }\end{array}$ & $\begin{array}{l}1047(68.9) \\
473(31.1)\end{array}$ & $\begin{array}{l}1839(84.8) \\
329(15.2)\end{array}$ & $<0.001 *$ \\
\hline $\begin{array}{l}\text { Marital status, } \mathrm{N}(\%) \\
\text { Unmarried } \\
\text { Married } \\
\text { CAT, mean (SD) }\end{array}$ & $\begin{array}{l}13(0.9) \\
1507(99.1) \\
15.3(6.3)\end{array}$ & $\begin{array}{l}17(0.8) \\
2151(99.2) \\
16.0(6.7)\end{array}$ & $\begin{array}{l}0.813 \\
0.003 *\end{array}$ \\
\hline $\begin{array}{l}\mathrm{mMRC}, \mathrm{N}(\%) \\
0-1 \\
2-4\end{array}$ & $\begin{array}{l}583(38.4) \\
937(61.6)\end{array}$ & $\begin{array}{l}618(28.5) \\
1550(71.5)\end{array}$ & $<0.001^{*}$ \\
\hline $\begin{array}{l}\text { Previous exacerbations, } \mathrm{N}(\%) \\
\quad<2 \\
\geq 2\end{array}$ & $\begin{array}{l}1075(70.7) \\
445(29.3)\end{array}$ & $\begin{array}{l}1268(58.5) \\
900(41.5)\end{array}$ & $<0.001 *$ \\
\hline 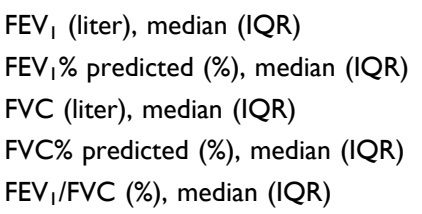 & $\begin{array}{l}1.10(0.74) \\
43.2(27.2) \\
2.46(1.07) \\
63.6(25.2) \\
46.5(18.4)\end{array}$ & $\begin{array}{l}1.10(0.76) \\
43.4(28.1) \\
2.50(0.94) \\
68.4(20.7) \\
45.6(18.3)\end{array}$ & $\begin{array}{l}0.735 \\
0.928 \\
0.414 \\
0.191 \\
0.064\end{array}$ \\
\hline $\begin{array}{l}\text { ABCD group, } N(\%) \\
\text { A } \\
\text { B } \\
\text { C } \\
\text { D }\end{array}$ & $\begin{array}{l}238(15.6) \\
837(55.1) \\
48(3.2) \\
397(26.1)\end{array}$ & $\begin{array}{l}292(13.5) \\
976(45.0) \\
100(4.6) \\
800(36.9)\end{array}$ & $<0.00 I^{*}$ \\
\hline $\begin{array}{l}\text { LAMA, N (\%) } \\
\text { ICS+LABA, N (\%) } \\
\text { ICS+LABA+LAMA, N (\%) }\end{array}$ & $\begin{array}{l}414(27.2) \\
265(17.4) \\
597(39.3)\end{array}$ & $\begin{array}{l}660(30.4) \\
342(15.8) \\
1001(46.2)\end{array}$ & $\begin{array}{l}0.035^{*} \\
0.191 \\
<0.001 *\end{array}$ \\
\hline
\end{tabular}

Notes: Categorical variables are summarized by frequencies, and statistical significance was determined by the Chi-square test. Continuous variables are described by mean \pm standard deviation (SD) or median \pm interquartile range (IQR), and statistical significance was determined by Student's $t$-test or Mann-Whitney $U$-test. $* P<0.05$. Abbreviations: BMI, body mass index; CAT, COPD Assessment Test; FEV , forced expiratory volume in Isecond; FVC, forced vital capacity; ICS, inhaled steroids; mMRC, modified Medical Research Council scale; LABA, long-acting $\beta_{2}$ agonists; LAMA, long-acting muscarinic antagonists.

with other risk factors remained higher than those in the tobacco only group.

After risk factor assessment, nonsmokers with COPD were classified into three groups: 423 patients were exposed to biomass smoke alone, 527 patients were exposed to occupational exposures alone, and 545 patients were exposed to other risk factors except tobacco, biomass smoke, and occupational exposure. Significant differences among the groups were observed. The percentage of females was higher in the biomass alone group than in the tobacco alone, the occupational exposure alone, and the other groups. We found that patients in the biomass 
alone group were the oldest, had the lowest educational level, and had the highest CAT scores $(17.5 \pm 6.3$ vs $15.3 \pm$ 6.3, $15.2 \pm 6.3$, and $16.3 \pm 6.6$, respectively, $P<0.05$ for each comparison, Table 3 ). Most of the components of the
CAT scores showed elevated levels in the biomass alone group compared with the other groups (Figure 2). In addition, patients in the tobacco alone group were less likely to develop more severe dyspnea $(61.6 \%$ vs $70.8 \%$ and

Table 3 Comparison of the Characteristics in the Subgroups of COPD Patients Exposed to Tobacco Alone, Biomass Smoke Alone, Occupational Exposure Alone, and Other Risk Factors

\begin{tabular}{|c|c|c|c|c|}
\hline & $\begin{array}{l}\text { Tobacco Alone } \\
(n=1520)\end{array}$ & $\begin{array}{l}\text { Biomass Smoke Alone } \\
(n=423)\end{array}$ & $\begin{array}{l}\text { Occupational } \\
\text { Exposure Alone } \\
(n=527)\end{array}$ & $\begin{array}{l}\text { Other Risk } \\
\text { Factors } \\
(n=545)\end{array}$ \\
\hline Age (years), mean (SD) & $64.6(8.2)$ & $65.8(8.5)^{\mathrm{a}}$ & $58.3(8.9)^{a, b}$ & $63.1(10.1)^{a, b, c}$ \\
\hline \multicolumn{5}{|l|}{ Sex, N (\%) } \\
\hline Male & I 498 (98.6) & $129(30.5)^{a}$ & $454(86.1)^{a, b}$ & $302(55.4)^{a, b, c}$ \\
\hline Female & $22(1.4)$ & $294(69.5)^{a}$ & $73(13.9)^{a, b}$ & $243(44.6)^{a, b, c}$ \\
\hline \multicolumn{5}{|l|}{ BMI (kg/m2), N (\%) } \\
\hline$<24$ & $1024(67.4)$ & $290(68.6)$ & $362(68.7)$ & $353(64.8)$ \\
\hline$\geq 24$ & $496(32.6)$ & $133(3 \mid .4)$ & $165(31.3)$ & $192(35.2)$ \\
\hline \multicolumn{5}{|l|}{ Education, N (\%) } \\
\hline Middle school or less & $1047(68.9)$ & $399(94.1)^{\mathrm{a}}$ & $465(88.2)^{a, b}$ & $419(76.9)^{a, b, c}$ \\
\hline High school or upper & $473(31.1)$ & $25(5.9)^{\mathrm{a}}$ & $62(11.8)^{a, b}$ & $126(23.1)^{a, b, c}$ \\
\hline \multicolumn{5}{|l|}{ Marital status, N (\%) } \\
\hline Unmarried & $13(0.9)$ & $2(0.5)$ & $7(1.3)$ & $4(0.7)$ \\
\hline Married & I507 (99.1) & 421 (99.5) & $520(98.7)$ & $54 I(99.3)$ \\
\hline CAT, mean (SD) & $15.3(6.3)$ & $17.5(6.3)^{a}$ & $15.2(6.3)^{b}$ & $16.3(6.6)^{a, b, c}$ \\
\hline \multicolumn{5}{|l|}{ mMRC, N (\%) } \\
\hline $0-1$ & $583(38.4)$ & $109(25.8)^{a}$ & $154(29.2)^{a}$ & $214(39.3)^{b, c}$ \\
\hline $2-4$ & $937(61.6)$ & $314(74.2)^{a}$ & $373(70.8)^{a}$ & $331(60.7)^{b, c}$ \\
\hline \multicolumn{5}{|l|}{ Previous exacerbations, N (\%) } \\
\hline$<2$ & $1075(70.7)$ & $233(55.1)^{\mathrm{a}}$ & $275(52.2)^{a}$ & $408(74.9)^{b, c}$ \\
\hline$\geq 2$ & $445(29.3)$ & $190(44.9)^{\mathrm{a}}$ & $252(47.8)^{a}$ & $137(25.1)^{b, c}$ \\
\hline $\mathrm{FEV}_{\text {, (liter), median (IQR) }}$ & $1.10(0.74)$ & $0.87(0.5 \mathrm{I})^{\mathrm{a}}$ & $1.22(0.83)^{a, b}$ & $0.96(0.63)$ a,b,c \\
\hline $\begin{array}{l}\mathrm{FEV}_{1} \% \text { predicted }(\%) \\
\text { median (IQR) }\end{array}$ & $43.2(27.2)$ & $46.5(24.9)^{a}$ & $46.3(27.4)^{\mathrm{a}}$ & $47.1(27.5)^{\mathrm{a}}$ \\
\hline FVC (liter), median (IQR) & $2.46(1.07)$ & $1.82(0.86)^{a}$ & $2.54(1.09)^{b}$ & $2.00(0.94)^{a, b, c}$ \\
\hline FVC\% predicted (\%), median (IQR) & $63.6(25.2)$ & $68.8(31.2)$ & $80.5(25.8)^{a}$ & $68.1(36.4)$ \\
\hline $\mathrm{FEV}_{\mathrm{I}} / \mathrm{FVC}(\%)$, median $(\mathrm{IQR})$ & $46.5(18.4)$ & $48.9(16.9)^{\mathrm{a}}$ & $51.6(17.4)^{a}$ & $50.5(18.5)^{a}$ \\
\hline \multicolumn{5}{|l|}{$A B C D$ group, $N(\%)$} \\
\hline A & $238(15.6)$ & $30(7.1)^{\mathrm{a}}$ & $69(13.1)^{b}$ & $76(13.9)^{b}$ \\
\hline B & $837(55.1)$ & $203(48.0)$ & $206(39.1)^{b}$ & $332(60.9)^{b, c}$ \\
\hline $\mathrm{C}$ & $48(3.2)$ & $13(3.1)$ & $29(5.5)^{f}$ & $8(1.5)$ \\
\hline $\mathrm{D}$ & $397(26.1)$ & $177(41.8)^{a}$ & $223(42.3)^{a}$ & $129(23.7))^{b, c}$ \\
\hline LAMA, N (\%) & $4 \mid 4(27.2)$ & $129(30.5)$ & $183(34.7)^{\mathrm{a}}$ & $122(22.4)^{b, c}$ \\
\hline ICS+LABA, N (\%) & $265(17.4)$ & $50(11.8)^{a}$ & $68(12.9)$ & $101(18.5)^{a, b}$ \\
\hline ICS+LABA+LAMA, N (\%) & $597(39.3)$ & $218(51.5)^{a}$ & $229(43.5)$ & $212(38.9)^{b}$ \\
\hline
\end{tabular}

Notes: Categorical variables are summarized by frequencies, and statistical significance was determined by the Chi-square or Fisher's test. Continuous variables are described by mean \pm standard deviation (SD) or median \pm interquartile range (IQR), and statistical significance was determined by one-way ANOVA or the Kruskal-Wallis test. ${ }^{a} P<0.05$ vs tobacco alone; ${ }^{b} P<0.05$ vs biomass smoke alone; ${ }^{c} P<0.05$ vs occupational exposure alone;

Abbreviations: BMI, body mass index; CAT, COPD Assessment Test; FEV , forced expiratory volume in I second; FVC, forced vital capacity; ICS, inhaled steroids; mMRC, modified Medical Research Council scale; LABA, long-acting $\beta 2$ agonists; LAMA, long-acting muscarinic antagonists. 
Table 4 Results are from Generalized Linear Mixed Models for the Association Between COPD Patients Exposed to Tobacco Alone and Tobacco Combined with Other Risk Factors

\begin{tabular}{|l|l|l|}
\hline \multirow{2}{*}{ Outcome } & \multicolumn{2}{|l|}{$\begin{array}{l}\text { Tobacco Alone vs Tobacco Combined } \\
\text { with Other Risk Factors }\end{array}$} \\
\cline { 2 - 3 } & OR $(95 \%$ CI) & P value \\
\hline CAT & $1.78(I .16,2.73)$ & $0.008^{*}$ \\
mMRC & $1.58(1.36,1.82)$ & $<0.00 I^{*}$ \\
Previous exacerbations & $1.66(I .44, I .92)$ & $<0.00 I^{*}$ \\
FEV $\%$ predicted & $1.48(0.42,5.20)$ & 0.534 \\
LAMA & $1.09(0.93,1.28)$ & 0.270 \\
ICS+LABA & $1.08(0.90,1.29)$ & 0.396 \\
ICS+LABA+LAMA & $1.03(0.88, I .21)$ & 0.671 \\
\hline
\end{tabular}

Notes: Data were adjusted for the potential confounders of sex, age, education level, body mass index, and marital status. OR denotes odds ratio and $\mathrm{Cl}$ denotes confidence interval. $* P<0.05$.

Abbreviations: CAT, COPD Assessment Test; mMRC, modified Medical Research Council scale.

$74.2 \%$, respectively, $P<0.05$ for both comparisons) and to have lower exacerbation ratios $(29.3 \%$ vs $47.8 \%$ and $44.9 \%$, respectively, $P<0.05$ for both comparisons) than patients in the occupational exposure alone and biomass alone groups. We developed a generalized linear mixed model that controlled for sex, age, BMI, marital status, and education level. We found that even after these potential confounders were accounted for, patients in the biomass alone group had higher CAT and mMRC scores and more frequent exacerbations than patients in the tobacco alone group ( $P=0.001, P=0.030$, and $P=0.010$, respectively, Table 5). We also noted persistent differences in mMRC scores and acute exacerbation frequency between the biomass smoke alone group and the other group $(P=0.002$ and $P<0.001$, respectively); between the tobacco alone group and the occupational exposure alone group $(P<0.001$ and $P<0.001$, respectively); and between the occupational exposure alone group and the other group ( $P=0.001$ and $P<0.001$, respectively).

\section{Discussion}

To the best of our knowledge, this is the study with the largest sample to present and compare the characteristics of COPD patients exposed to different risk factors. Herein, we report the demographic features and clinical symptoms of predefined COPD groups in China. In general, COPD patients who are exposed to tobacco plus other risk factors present with more severe clinical symptoms than patients who are exposed to tobacco alone. This difference remained strong even after adjustment for potential confounders. Furthermore, COPD patients exposed to biomass smoke were more likely to be female, older, and more symptomatic, whereas COPD patients exposed to occupational exposures were significantly
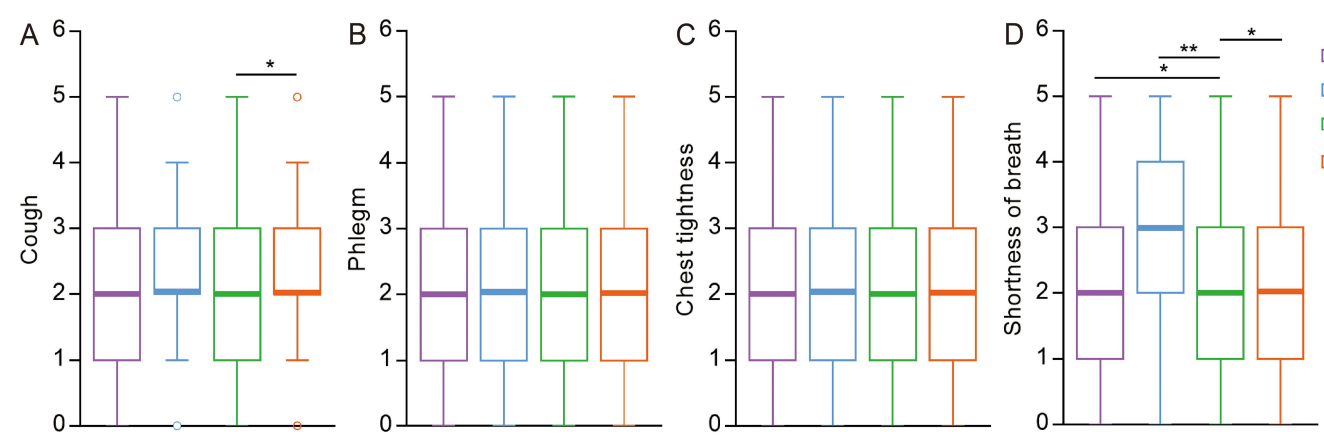

$\square$ Tobacco alone

$\square$ Biomass smoke alone

$\square$ Occupational exposure alone

Other risk factors
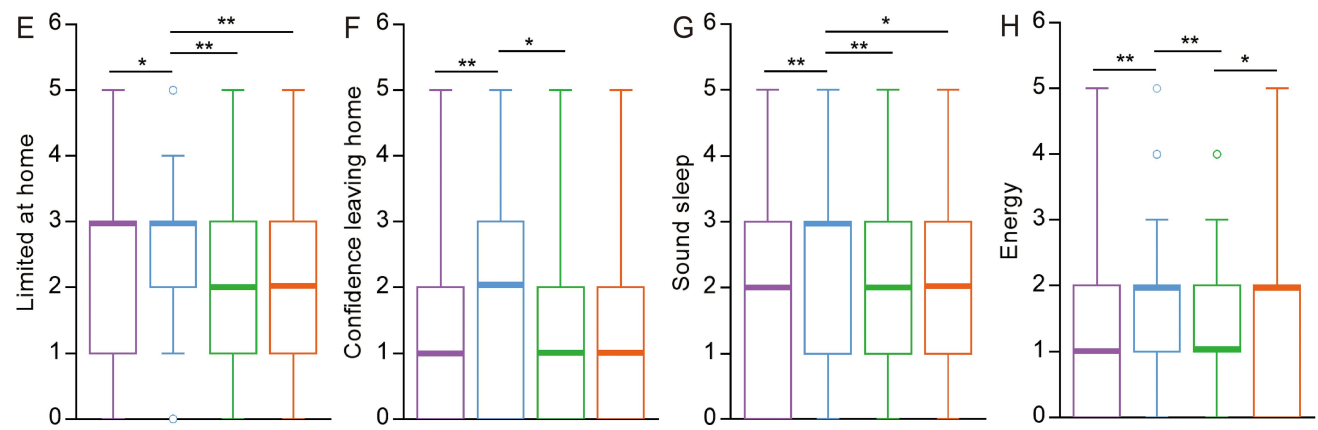

Figure 2 CAT component scores in COPD patients exposed to tobacco alone, biomass smoke alone, occupational exposure alone, and other risk factors.

Note: (A) Cough. (B) Phlegm. (C) Chest tightness. (D) Shortness of breath. (E) Limited at home. (F) Confidence leaving home. (G) Sound sleep. (H) Energy. $* P<0.05$; $* * P$ $<0.001$. 
Table 5 Results are from Generalized Linear Mixed Models for the Association Among COPD Patients Exposed to Tobacco Alone, Biomass Smoke Alone, Occupational Exposure Alone, and Other Risk Factors

\begin{tabular}{|c|c|c|c|c|}
\hline \multirow[t]{2}{*}{ Outcome } & \multicolumn{2}{|c|}{ Biomass Smoke Alone vs Tobacco Alone } & \multicolumn{2}{|c|}{ Biomass Smoke Alone vs Occupational Exposure Alone } \\
\hline & OR $(95 \% \mathrm{Cl})$ & $P$ value & OR $(95 \% \mathrm{Cl})$ & $P$ value \\
\hline CAT & $5.41(1.91,15.33)$ & $0.001 *$ & $2.64(0.94,7.38)$ & 0.064 \\
\hline $\mathrm{mMRC}$ & $1.53(1.04,2.23)$ & $0.030 *$ & $1.07(0.73,1.55)$ & 0.745 \\
\hline Previous exacerbations & $1.58(1.12,2.23)$ & $0.010^{*}$ & $1.07(0.76,1.49)$ & 0.700 \\
\hline $\mathrm{FEV}, \%$ predicted & $0.72(0.03,15.32)$ & 0.832 & $0.14(0.01,2.34)$ & 0.172 \\
\hline LAMA & $1.17(0.93,1.48)$ & 0.187 & $0.83(0.63,1.08)$ & 0.178 \\
\hline ICS+LABA & $0.34(0.19,0.58)$ & $<0.001 *$ & $0.69(0.40,1.18)$ & 0.179 \\
\hline ICS+LABA+LAMA & $1.58(1.09,2.29)$ & $0.016 *$ & $1.36(0.93,1.97)$ & 0.107 \\
\hline \multirow[t]{2}{*}{ Outcome } & \multicolumn{2}{|c|}{ Biomass smoke alone vs Other risk factors } & \multicolumn{2}{|c|}{ Tobacco alone vs Occupational exposure alone } \\
\hline & OR $(95 \% \mathrm{CI})$ & $P$ value & OR $(95 \% \mathrm{Cl})$ & $P$ value \\
\hline CAT & $2.22(0.94,5.26)$ & 0.070 & $1.05(0.54,2.04)$ & 0.897 \\
\hline $\mathrm{mMRC}$ & $1.61(1.19,2.16)$ & $0.002 *$ & $0.45(0.35,0.59)$ & $<0.00 I^{*}$ \\
\hline Previous exacerbations & $2.38(1.78,3.19)$ & $<0.001 *$ & $0.43(0.34,0.54)$ & $<0.00 I^{*}$ \\
\hline $\mathrm{FEV}, \%$ predicted & $4.53(0.40,50.95)$ & 0.221 & $11.59(1.56,86.45)$ & $0.017 *$ \\
\hline LAMA & $1.06(0.77,1.45)$ & 0.725 & $0.83(0.65,1.06)$ & 0.132 \\
\hline ICS+LABA & $0.38(0.26,0.57)$ & $<0.001 *$ & $1.16(0.85,1.58)$ & 0.334 \\
\hline ICS+LABA+LAMA & $1.02(0.76,1.36)$ & 0.893 & $0.85(0.66,1.08)$ & 0.176 \\
\hline \multirow[t]{2}{*}{ Outcome } & \multicolumn{2}{|c|}{ Tobacco alone vs Other risk factors } & \multicolumn{2}{|c|}{ Occupational exposure alone vs Other risk factors } \\
\hline & OR $(95 \% \mathrm{Cl})$ & $P$ value & OR $(95 \% \mathrm{Cl})$ & $P$ value \\
\hline CAT & $0.26(0.13,0.53)$ & $0.003 *$ & $2.09(0.92,4.78)$ & 0.080 \\
\hline $\mathrm{mMRC}$ & $1.03(0.80,1.33)$ & 0.795 & $1.61(1.21,2.14)$ & $0.001 *$ \\
\hline Previous exacerbations & $1.23(0.99,1.54)$ & 0.066 & $2.58(1.93,3.44)$ & $<0.00 I^{*}$ \\
\hline $\mathrm{FEV}, \%$ predicted & $0.79(0.09,6.98)$ & 0.830 & $10.79(0.95,81.87)$ & 0.054 \\
\hline LAMA & $1.68(1.22,2.20)$ & 0.313 & $2.00(1.45,2.77)$ & $<0.00 I^{*}$ \\
\hline ICS+LABA & $0.8 \mathrm{I}(0.58,1.14)$ & 0.230 & $0.78(0.54,1.13)$ & 0.190 \\
\hline ICS+LABA+LAMA & $0.8 \mathrm{I}(0.62,1.03)$ & 0.090 & $1.19(0.88,1.62)$ & 0.247 \\
\hline
\end{tabular}

Notes: Data were adjusted for the potential confounders of sex, age, education level, body mass index, and marital status. OR denotes odds ratio and Cl denotes confidence interval. $* P<0.05$.

Abbreviations: CAT, COPD Assessment Test; mMRC, modified Medical Research Council scale.

younger. Therefore, this study confirmed that there are differences in the characteristics of COPD patients exposed to tobacco smoking, biomass smoke, and occupational sources.

It should be highlighted that COPD patients were assigned to the five different subgroups based on exposure to various risk factors in this study. We found that smokers constituted two-thirds of all COPD cases. The same pattern was evident in other studies. ${ }^{19,20}$ A previous study revealed that tobacco smoking accounted for $35 \%$ of the population's attributable risk for COPD, and the risk increased with packyears. ${ }^{2}$ In addition, we noticed that there were many nonsmokers and some smokers exposed to other risk factors at the same time, which may, in turn, affect the symptomatology of patients. Despite the high burden of smokers with COPD, we must pay much more attention to other risk factors.
A growing number of studies have provided evidence that the considerable proportion of patients with COPD was significantly associated with occupational exposures. ${ }^{21-23}$ Recent evidence from a survey conducted in the United States illustrated that a quarter to half of nonsmoking COPD cases could be attributed to occupational exposures. ${ }^{24}$ The harm caused by occupational dust or gas exposure was more severe in developing countries than in developed countries. However, it is not easy to determine the total amount of occupational exposure, the length of exposure time, and the concentration or type of occupational pollutants, which has made progress in relevant research relatively slow. To the best of our knowledge, China has one of the highest numbers of occupational cases in the world. $^{25}$ Although there were minimal differences in 
respiratory symptoms between COPD patients with occupational exposure and smokers alone, there were clear differences in baseline patient characteristics between the groups. We found that COPD patients who were suffering from occupational exposure were the youngest, which is not consistent with the findings of previous studies conducted abroad. ${ }^{7}$ One explanation could be related to China's national conditions. Protective measures have been implemented in China to prevent and control occupational diseases, which requires workers exposed to occupational disease hazards to have free medical examinations on schedule. This law makes it possible for more patients aged 40 to 59 years who are engaged in occupational disease-related work to see a doctor, increasing the probability of a diagnosis of COPD. In contrast, smokers with COPD generally consider respiratory symptoms to be associated with the cigarette smoking itself and thus avoid seeking medical attention. Moreover, patients exposed to biomass smoke often live far away from the hospital and under poor economic conditions. Sometimes, these patients do not come to the hospital until they have obvious symptoms.

Much less biomass fuel is used in developed countries such as Spain. ${ }^{26}$ However, the frequency of biomass smoke exposure was high in our study. Available epidemiological studies demonstrate that biomass smoke is an important cause of COPD. ${ }^{27,28}$ When air pollution from biomass is reduced, the prognosis and lung function of COPD patients are improved. ${ }^{29,30}$ Another survey indicates that household-level interventions for biomass smoke reduction have benefits for respiratory health. ${ }^{31}$ In particular, poor kitchen ventilation in rural or remote areas of China with impoverished living conditions leads to very high biomass smoke concentrations. Additionally, strong sex differences were observed between COPD patients exposed to biomass smoke alone and other COPD patients. Women spend the majority of their time cooking food using biomass fuels in China due to the influence of traditional feudal ideals. Therefore, we revealed that women have a greater risk of being exposed to biomass smoke, which was consistent with the results of earlier research. ${ }^{32}$ In our models, we adjusted for sex and age at baseline, and we discovered that COPD patients with biomass smoke exposure still had more serious respiratory symptoms. Biomass smoke exposure was associated with small airway obstruction, and more air trapping was demonstrated in a cross-sectional study ${ }^{33}$ and recently in another study from India. ${ }^{13}$ Biomass smoke has also been shown to cause more hypoxemia. ${ }^{34}$ All these findings may explain why COPD patients with biomass smoke exposure were more symptomatic.

In our study, patients who were exposed to tobacco combined with other risk factors were estimated to account for $58.8 \%$ of all smokers with COPD, and $98.8 \%$ of them were male, which were similar to the results for patients exposed to tobacco alone. This is presumably because of the relatively high proportion of adult men in China who smoke cigarettes. There have been few studies on the characteristics of COPD patients with combined exposure to tobacco and other risk factors to date. One survey reported that COPD caused by tobacco plus other inhalants led to greater impairment of quality of life. ${ }^{26}$ Our work demonstrated that patients exposed to tobacco plus other risk factors had worse respiratory symptoms and more frequent exacerbations than those who were exposed to tobacco alone. This finding suggests that combined exposure to tobacco plus other risk factors has a noxious additive effect in COPD patients and could result in more serious COPD symptoms.

With rapid socioeconomic and industrial development, ambient air pollution has become a major public health crisis around the world. ${ }^{35}$ Previous studies suggested that exposure to higher PM2.5 concentrations was closely associated with an increased prevalence of COPD and decreased respiratory function, ${ }^{36}$ especially in young adults. ${ }^{3}$ Additionally, recurrent respiratory tract infections in early childhood, chronic cough during childhood, tobacco exposure in utero, low birth weight, preterm birth, and genetic factors were significantly associated with COPD. ${ }^{37-40}$ Our study revealed that approximately $10 \%$ of COPD patients were exposed to these unusual risk factors. We identified that these COPD patients have obvious respiratory symptoms and create a heavy disease burden. In a follow-up study, we will further classify other uncommon risk factors in the Chinese population and compare their clinical symptoms.

This study has several limitations. We employed no stratification of exposure to different risk factors, which would have provided extra information. Another limitation was that the participants in this study were mainly from Hunan Province and Guangxi Zhuang Autonomous Region (central-south region of China). To reduce the bias associated with convenient samples, we collected as many patients as possible.

\section{Conclusion}

In summary, in this large-sample study, we observed and compared the demographic features and clinical characteristics 
of COPD patients exposed to different risk factors in China. There were significant demographic and clinical differences among COPD patients with tobacco smoking, biomass smoke, and occupational exposure. The information provided here can act as a guide to warn doctors of the effects of these risk factors and to encourage them to take effective action to improve the targeted prevention of COPD.

\section{Acknowledgments}

The authors would like to thank the staff of all the participating hospitals for their cooperation in collecting the study data. This work was supported by the National Natural Science Foundation (Grant number 81770046) and the Innovation Fund Designated for Graduate Students of Hunan Province (Grant number CX20190073).

\section{Disclosure}

The authors report no conflicts of interest in this work.

\section{References}

1. Singh D, Agusti A, Anzueto A, et al. Global strategy for the diagnosis, management, and prevention of Chronic Obstructive Lung Disease: the GOLD science committee report 2019. Eur Respir J. 2019;53:5.

2. Global, regional, and national incidence, prevalence, and years lived with disability for 310 diseases and injuries, 1990-2015: a systematic analysis for the Global Burden of Disease Study 2015. Lancet (London, England). 2016;388(10053):1545-1602.

3. Wang $\mathrm{C}, \mathrm{Xu} \mathrm{J}$, Yang L, et al. Prevalence and risk factors of chronic obstructive pulmonary disease in China (the China Pulmonary Health $[\mathrm{CPH}]$ study): a national cross-sectional study. Lancet (London, England). 2018;391(10131):1706-1717. doi:10.1016/S0140-6736(18)30841-9

4. Capistrano SJ, van Reyk D, Chen H, Oliver BG. Evidence of biomass smoke exposure as a causative factor for the development of COPD. Toxics. 2017;5:4. doi:10.3390/toxics5040036

5. Assad NA, Balmes J, Mehta S, Cheema U, Sood A. Chronic obstructive pulmonary disease secondary to household air pollution. Semin Respir Crit Care Med. 2015;36(3):408-421. doi:10.1055/s-0035-1554846

6. Terzikhan N, Verhamme KM, Hofman A, Stricker BH, Brusselle GG, Lahousse L. Prevalence and incidence of COPD in smokers and non-smokers: the Rotterdam Study. Eur J Epidemiol. 2016;31 (8):785-792. doi:10.1007/s10654-016-0132-z

7. Hagstad S, Backman H, Bjerg A, et al. Prevalence and risk factors of COPD among never-smokers in two areas of Sweden - Occupational exposure to gas, dust or fumes is an important risk factor. Respir Med. 2015;109(11):1439-1445. doi:10.1016/j.rmed.2015.09.012

8. Szram J, Schofield SJ, Cosgrove MP, Cullinan P. Welding, longitudinal lung function decline and chronic respiratory symptoms: a systematic review of cohort studies. Eur Respir J. 2013;42 (5):1186-1193. doi:10.1183/09031936.00206011

9. Kuempel ED, Wheeler MW, Smith RJ, Vallyathan V, Green FH. Contributions of dust exposure and cigarette smoking to emphysema severity in coal miners in the United States. Am J Respir Crit Care Med. 2009;180(3):257-264. doi:10.1164/rccm.200806-840OC

10. Marchetti N, Garshick E, Kinney GL, et al. Association between occupational exposure and lung function, respiratory symptoms, and high-resolution computed tomography imaging in COPDGene. Am $J$ Respir Crit Care Med. 2014;190(7):756-762. doi:10.1164/rccm. 201403-0493OC
11. Rivera RM, Cosio MG, Ghezzo H, Salazar M, Perez-Padilla R. Comparison of lung morphology in COPD secondary to cigarette and biomass smoke. Int J Tuberculosis Lung Dis. 2008;12(8):972-977.

12. Golpe R, Martin-Robles I, Sanjuan-Lopez P, et al. Differences in systemic inflammation between cigarette and biomass smoke-induced COPD. Int J Chron Obstruct Pulmon Dis. 2017;12:2639-2646. doi:10.2147/COPD.S141068

13. Salvi SS, Brashier BB, Londhe J, et al. Phenotypic comparison between smoking and non-smoking chronic obstructive pulmonary disease. Respir Res. 2020;21(1):50. doi:10.1186/s12931-020-1310-9

14. Zhao D, Zhou Y, Jiang C, Zhao Z, He F, Ran P. Small airway disease: a different phenotype of early stage COPD associated with biomass smoke exposure. Respirology (Carlton, Vic). 2018;23(2):198-205. doi: $10.1111 /$ resp. 13176

15. Vinnikov D, Semizhon S, Rybina T, Savich L, Scherbitsky V, Manichev I. Occupation and chronic obstructive pulmonary disease in Minsk tractor plant workers. Am J Ind Med. 2017;60 (12):1049-1055. doi:10.1002/ajim.22776

16. Jones PW, Harding G, Berry P, Wiklund I, Chen WH, Kline Leidy N. Development and first validation of the COPD Assessment Test. Eur Respir J. 2009;34(3):648-654. doi:10.1183/09031936.00102509

17. Woodruff PG, Barr RG, Bleecker E, et al. Clinical significance of symptoms in smokers with preserved pulmonary function. $N$ Engl J Med. 2016;374(19):1811-1821. doi:10.1056/NEJMoa1505971

18. Bestall JC, Paul EA, Garrod R, Garnham R, Jones PW, Wedzicha JA. Usefulness of the Medical Research Council (MRC) dyspnoea scale as a measure of disability in patients with chronic obstructive pulmonary disease. Thorax. 1999;54(7):581-586.

19. Zhang J, Lin XF, Bai CX. Comparison of clinical features between non-smokers with COPD and smokers with COPD: a retrospective observational study. Int J Chron Obstruct Pulmon Dis. 2014;9:57-63. doi:10.2147/COPD.S52416

20. Lamprecht B, McBurnie MA, Vollmer WM, et al. COPD in never smokers: results from the population-based burden of obstructive lung disease study. Chest. 2011;139(4):752-763. doi:10.1378/chest.10-1253

21. Alblin M, Gustavsson P. A silent epidemic: occupational exposure limits are insufficiently protecting individual worker health. Scand $J$ Work Environ Health. 2020;46(1):110-112.

22. Sadhra S, Kurmi OP, Sadhra SS, Lam KB, Ayres JG. Occupational COPD and job exposure matrices: a systematic review and meta-analysis. Int J Chron Obstruct Pulmon Dis. 2017;12:725-734. doi: $10.2147 /$ COPD.S125980

23. Lytras T, Kogevinas M, Kromhout H, et al. Occupational exposures and 20-year incidence of COPD: the European community respiratory health survey. Thorax. 2018;73(11):1008-1015. doi:10.1136/ thoraxjnl-2017-211158

24. Syamlal G, Doney B, Mazurek JM. Chronic Obstructive Pulmonary Disease prevalence among adults who have never smoked, by industry and occupation - United States, 2013-2017. MMWR Morb Mortal Wkly Rep. 2019;68(13):303-307. doi:10.15585/mmwr.mm6813a2

25. Peng Y, Li X, Cai S, et al. Prevalence and characteristics of COPD among pneumoconiosis patients at an occupational disease prevention institute: a cross-sectional study. BMC Pulm Med. 2018;18 (1):22. doi:10.1186/s12890-018-0581-0

26. Lopez-Campos JL, Fernandez-Villar A, Calero-Acuna C, et al. Occupational and biomass exposure in chronic obstructive pulmonary disease: results of a cross-sectional analysis of the on-sint study. Arch Bronconeumol. 2017;53(1):7-12. doi:10.1016/j.arbr.2016.06.022

27. Zhu B, Wang Y, Ming J, Chen W, Zhang L. Disease burden of COPD in China: a systematic review. Int J Chron Obstruct Pulmon Dis. 2018;13:1353-1364. doi:10.2147/COPD.S161555

28. Bajpai J, Kant S, Bajaj DK, Pradhan A, Srivastava K, Pandey AK. Clinical, demographic and radiological profile of smoker COPD versus nonsmoker COPD patients at a tertiary care center in North India. J Family Med Prim Care. 2019;8(7):2364-2368. doi:10.4103/ jfmpc.jfmpc_347_19 
29. Zhou Y, Zou Y, Li X, et al. Lung function and incidence of chronic obstructive pulmonary disease after improved cooking fuels and kitchen ventilation: a 9-year prospective cohort study. PLoS Med. 2014;11(3):e1001621. doi:10.1371/journal.pmed.1001621

30. Johnston FH, Hanigan IC, Henderson SB, Morgan GG. Evaluation of interventions to reduce air pollution from biomass smoke on mortality in Launceston, Australia: retrospective analysis of daily mortality, 1994-2007. BMJ (Clinical Research Ed). 2013;346:e8446.

31. Saleh S, Shepherd W, Jewell C, et al. Air pollution interventions and respiratory health: a systematic review. Int $j$ Tuberculosis Lung Dis. 2020;24(2):150-164. doi:10.5588/ijtld.19.0417

32. Olloquequi J, Jaime S, Parra V, et al. Comparative analysis of COPD associated with tobacco smoking, biomass smoke exposure or both Respir Res. 2018;19(1):13. doi:10.1186/s12931-018-0718-y

33. Camp PG, Ramirez-Venegas A, Sansores RH, et al. COPD phenotypes in biomass smoke- versus tobacco smoke-exposed Mexican women. Eur Respir J. 2014;43(3):725-734. doi:10.1183/09031936. 00206112

34. Meneghini AC, Koenigkam-Santos M, Pereira MC, et al. Biomass smoke COPD has less tomographic abnormalities but worse hypoxemia compared with tobacco COPD. Braz J Med Biol Res/Revista Brasileira de Pesquisas Medicas e Biologicas. 2019;52(5):e8233. doi:10.1590/1414-431x20198233
35. Schikowski T, Adam M, Marcon A, et al. Association of ambient air pollution with the prevalence and incidence of COPD. Eur Respir J. 2014;44(3):614-626. doi:10.1183/09031936.00132213

36. Liu S, Zhou Y, Liu S, et al. Association between exposure to ambient particulate matter and chronic obstructive pulmonary disease: results from a cross-sectional study in China. Thorax. 2017;72(9):788-795. doi:10.1136/thoraxjnl-2016-208910

37. van Meel ER, den Dekker HT, Elbert NJ, et al. A population-based prospective cohort study examining the influence of early-life respiratory tract infections on school-age lung function and asthma. Thorax. 2018;73(2):167-173. doi:10.1136/thoraxjnl-2017-210149

38. Savran O, Ulrik CS. Early life insults as determinants of chronic obstructive pulmonary disease in adult life. Int $J$ Chron Obstruct Pulmon Dis. 2018;13:683-693. doi:10.2147/COPD.S153555

39. Perret JL, Walters H, Johns D, et al. Mother's smoking and complex lung function of offspring in middle age: a cohort study from childhood. Respirology (Carlton, Vic). 2016;21(5):911-919. doi:10.1111/ resp. 12750

40. Kaczmarczyk K, Wiszomirska I, Szturmowicz M, Magiera A, Błażkiewicz M. Are preterm-born survivors at risk of long-term respiratory disease? Ther Adv Respir Dis. 2017;11(7):277-287. doi:10.1177/1753465817710595

\section{Publish your work in this journal}

The International Journal of COPD is an international, peer-reviewed journal of therapeutics and pharmacology focusing on concise rapid reporting of clinical studies and reviews in COPD. Special focus is given to the pathophysiological processes underlying the disease, intervention programs, patient focused education, and self management protocols. This journal is indexed on PubMed Central, MedLine and CAS. The manuscript management system is completely online and includes a very quick and fair peer-review system, which is all easy to use. Visit http://www.dovepress.com/testimonials.php to read real quotes from published authors. 